\section{A perspective on the current management of advanced colorectal cancer}

\author{
Mariaelena Casagrande ${ }^{\ddagger 1}$, Chiara Cremolini $\ddagger^{\ddagger 2}$, Fotios Loupakis ${ }^{2}$ \\ \& Giuseppe Aprile ${ }^{\star 1}$ \\ 'Department of Oncology, University \& General Hospital, Udine, Italy \\ 2Department of Oncology, University \& General Hospital. Pisa, Italy \\ *Author for correspondence: aprile.giuseppe@aoud.sanita.fvg.it \\ ‡Authors contributed equally
}

\section{World Congress on Gastrointestinal Cancers}

\section{3-6 July 2013, Barcelona, Spain}

Although the median survival of patients with advanced disease has progressively increased, colorectal cancer remains a major worldwide health problem, with approximately 1 million new cases and 500,000 deaths every year. At the 2013 World Congress on Gastrointestinal Cancers, investigators have recently dealt with many clinically relevant questions, such as the preferred biologic drug to be used upfront in patients with KRAS wild-type colorectal tumors, the optimal treatment intensity, the most suitable maintenance strategy, the need for deeper molecular information when using EGFR inhibitors and the use of antiangiogenic drugs in the elderly. Briefly summarizing all the news coming from this moving landscape, this article focuses on the results of the major randomized Phase III trials presented at the meeting.

Launched in 1999 as a small expert meeting called 'Perspective in Colorectal Cancer', over time this summer opening scientific appointment has changed its name (since 2004), gained the European Society of Medical Oncology sponsorship and the full endorsement of many scientific societies, remarkably improved its format and teaching value, and vastly enlarged its borders, yet still preserving the original commitment to educate and share research. Celebrating its 15 th anniversary this year, the renewed 'World Congress on Gastrointestinal Cancers' is now classified as a premium international forum focused on research and multidisciplinary management of digestive tract malignancies. This first-class international meeting was held between the 3rd and 6th July 2013 in Barcelona (Spain), a privileged colorful city on the northeast coast of Catalonia, Spain, where leading oncologists gathered with over 3000 cancer specialty professional attendees to share and discuss the latest advances in the field. The conference embraced all aspects of gastrointestinal cancer care, ranging from diagnostics to translational research and from multimodal upfront treatment to palliation. Under the auspices of a valuable scientific committee, the key 3-day scientific meeting had a very busy schedule, including plenary educational sessions, keynote lectures and oral presentations, as well as the exposition of nearly 300 posters and satellite symposia. In this short conference report, the authors summarize the hot-topic highlights on advanced colorectal cancers (CRCs), and offer the readers an independent, opinion-based overview of the most noteworthy data presented at the meeting that are likely to soon have an impact on real-world clinical practice.

\section{Upfront antiangiogenic therapy or EGFR inhibitors? First data on the head-to-head comparison of KRAS wild-type CRC patients}

Determining which targeted agent should be combined with first-line chemotherapy in KRAS wild-type patients is one of the most heated issues in colorectal oncology. The FIRE-3 trial, initially presented at the 2013 American Society of Clinical Oncology meeting, was the first Phase III randomized trial to provide results on this highly invoked head-to-head comparison [1]. The German trial randomized 592 KRAS wild-type CRC patients to upfront folinic acid, fluorouracil and irinotecan (FOLFIRI) plus either cetuximab or bevacizumab. Although the primary end point, which was the objective response rate (ORR), was not met $(62 \%$ in the FOLFIRI plus cetuximab arm vs $58 \%$ in the FOLFIRI plus bevacizumab arm; $\mathrm{p}=0.18$ ) and no differences in progression-free survival (PFS) were reported (hazard ratio [HR]: 1.06; 95\% CI: $0.88-1.26 ; \mathrm{p}=0.54)$, a significant
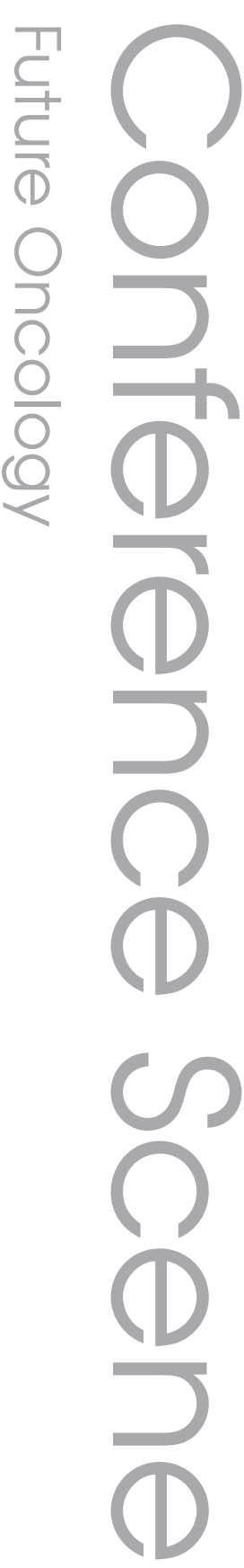

\section{Keywords}

- bevacizumab = colorectal cancer $=$ EGFR inhibitor - elderly = maintenance " neurotoxicity = NRAS

\section{Future : $\because$ fS}


advantage in overall survival (OS) was observed in favor of the anti-EGFR arm (HR: 0.77; 95\% CI: $0.62-0.96 ; \mathrm{p}=0.017$ ). In order to further explore and clarify these unexpected results, additional data on subsequent lines were eagerly awaited. According to Modest's presentation, disparities in second-line treatments are unlikely to explain the observed survival difference [2] because a similar proportion of patients received second-line treatments (78.5 vs $76.4 \%$ ). Moreover, $46.6 \%$ of patients who were initially exposed to cetuximab received bevacizumab, and $41.4 \%$ of patients initially treated with bevacizumab eventually received EGFR inhibitors. Consistently, a Phase II randomized PEAK trial of first-line folinic acid, fluorouracil (5-FU) and oxaliplatin (FOLFOX) plus bevacizumab versus FOLFOX plus panitumumab showed a trend toward improved OS in the anti-EGFR arm (HR: 0.72; 95\% CI: 0.47-1.11; $\mathrm{p}=0.14$ ) [3]. Indeed, the association of the response rate to EGFR inhibitors with the postprogression survival was advocated as a possible reason for success [4]. According to this theoretical model, the higher tumor shrinkage may result in a lower tumor load, as per the Response Evaluation Criteria In Solid Tumors, at the time of disease progression so that the benefit achieved in terms of response rate may influence the progression of patients' disease. While a similar confirmatory correlation analysis is being conducted in the FIRE-3 trial, survival results from a larger Intergroup Phase III trial (C80405), which aims to compare upfront bevacizumab and cetuximab in 2900 patients, are awaited.

\section{Use of EGFR inhibitors needs a deeper molecular selection}

As pointed out at the last American Society of Clinical Oncology meeting, an important achievement regarding the use of EGFR inhibitors is now well established: a more accurate molecular selection, beyond KRAS codon 12 and 13 mutational status, allows us to identify a wider population that would not benefit from such treatments [5]. Retrospective analyses of the first-line Phase III PRIME [6] and the Phase II PEAK [3] trials repeatedly showed that patients harboring rare $K R A S$ mutations in exon 3 and 4 , as well as NRAS exon 2, 3 and 4 mutations, do not benefit from the combination. Importantly, the addition of panitumumab to first-line FOLFOX might even be detrimental in CRC patients with $R A S$ mutations and should be carefully avoided. On the basis of these data, the EMA's Committee for Medicinal Products for Human Use has (promptly) amended the marketing authorization for panitumumab, limiting its use in 'all$R A S$ ' wild-type CRC patients. Since it has been highlighted how a well-characterized molecular profile may impact on the evidence-based decision-making process, a substantial shift in the selection of patients suitable for treatment with EGFR inhibitors is ongoing.

\section{Fine-tuning in the upfront treatment intensity}

Recent evidence supports the idea that the increased activity of an intensive upfront regimen may actually translate into a noteworthy survival benefit [7]. The intensity of the upfront chemotherapy-backbone is therefore also important in the targeted agent era. Two randomized trials, OLIVIA [8] and TRIBE [9], investigated the combination of folinic acid, 5-FU, oxaliplatin and irinotecan (FOLFOXIRI) with bevacizumab in different clinical settings, providing results in terms of outcome and safety. In the Phase II OLIVIA trial, 80 patients with unresectable metastases confined to the liver were randomized to FOLFOX plus bevacizumab or FOLFOXIRI plus bevacizumab. Patients allocated to the more intense regimen had a higher ORR ( 80.5 vs $61.5 \%$; $=0.061)$. Moreover, both overall resection rate (61.0 vs $48.7 \%$; $p=0.271$ ) and radical resection rate (48.8 vs $23.1 \%$; $\mathrm{p}=0.017)$ were higher in the FOLFOXIRI plus bevacizumab arm. In addition, substantial benefit in terms of PFS was noted (21 vs 11.6 months; $p=0.0002$ ), confirming the effectiveness of the combination for patients with liver-limited disease. In the Phase III TRIBE trial, 508 unresectable advanced CRC patients were randomized to receive up to 12 cycles of FOLFIRI plus bevacizumab or FOLFOXIRI plus bevacizumab, followed by maintenance 5-FU/leucovorin plus bevacizumab until disease progression. The primary end point was met: patients in the experimental arm achieved a significant benefit in median PFS (12.1 vs 9.7 months; HR: 0.77; 95\% CI: 0.64-0.93; $\mathrm{p}=0.006)$. Although the triplet provided a significantly higher ORR (65 vs $53 \% ; p=0.006$ ), the corresponding resection rate (15 vs $12 \%)$ was not improved. However, it should be noted that the study population was unselected for conversion to surgical resectability, since only $20 \%$ of enrolled patients had liver-limited disease. Moreover, a trend toward improved survival in the FOLFOXIRI plus bevacizumab arm was reported (31 vs 25.8 months; HR: 0.83 ; 95\% CI: 0.66-1.05; $p=0.125$ ), making the regimen an upfront treatment option 
to also be considered in a palliative setting, independently from a conversion intent.

\section{Elderly individuals with advanced CRC: could antiangiogenic drugs increase the treatment benefit?}

Until 2013, the use of antiangiogenic drugs in older people has been debated due to an unconvincing risk:benefit ratio [10]. In the Phase III AVEX trial, 280 elderly CRC patients (median age: 76 years) were randomized to receive firstline capecitabine $\left(2000 \mathrm{mg} / \mathrm{m}^{2}\right.$ daily $)$ with or without bevacizumab $(7.5 \mathrm{mg} / \mathrm{kg})$. The combination therapy significantly increased the ORR (19.3 vs 10\%) and median PFS (9.1 vs 5.1 months; HR: 0.53; 95\% CI: 0.41-0.69), and produced longer OS (20.7 vs 16.8 months; HR: 0.79; 95\% CI: 0.57-1.09) [11]. The agecohort breakdown presented at the meeting demonstrated that the benefit derived from the use of bevacizumab may even be increased for the oldest patients ( $\geq 80$ years), with the HR for PFS and OS being 0.36 (95\% CI: 0.19-0.71) and 0.62 (95\% CI: 19.7-12.6), respectively [12]. Treatment was well-tolerated overall, regardless of age. The efficacy of combining aflibercept with second-line FOLFIRI was assessed in 1226 patients enrolled in the Phase III VELOUR trial [13]. In the older patient cohort (438 patients aged $\geq 65$ years) the results were confirmed: compared with FOLFIRI and placebo, the combination of FOLFIRI and aflibercept produced significantly superior median PFS (6.6 vs 4.4 months; HR: 0.74; 95\% CI: 0.59-0.93) and longer median OS (12.6 vs 11.3 months; HR: 0.85 ; 95\% CI: 0.68-1.06), with a similar toxicity profile except for the increased incidence of dehydration $(\mathrm{p}=0.005)$ [14].

\section{The issue of maintenance}

Results of randomized trials (MACRO, DREAM and COIN-B) suggest the continuation of antiangiogenic therapy as maintenance therapy after first-line treatment [15-17]. In CAIRO-3, patients without disease progression after six cycles of capecitabine, oxaliplatin and bevacizumab were randomized to observation or maintenance with capecitabine plus bevacizumab [18]. In CAIRO-3, maintenance treatment was demonstrated as advantageous, significantly prolonging the time from randomization until first progression after observation or maintenance treatment ( 8.5 vs 4.1 months; HR: 0.44$)$, the time from randomization to progression upon reintroduction of the initial treatment (11.8 vs 10.5 months; HR: 0.81), and the OS (21.7 vs 18.2 months; HR: 0.87). With 262 advanced CRC patients enrolled, the aim of the Phase III SAKK 41/06 study was to demonstrate the noninferiority of continuing bevacizumab versus no treatment after 4-6 months of first-line treatment [19]. Although median PFS and OS were both longer for patients who received maintenance, the trial formally failed to demonstrate its primary end point, since the median time to progression did not differ significantly between the two arms (17.9 weeks for the maintenance arm vs 12.6 weeks for the observational arm; HR: 0.72; with a noninferiority limit HR of 0.727). While reasonable and clinically feasible, whether a maintenance therapy is needed for all patients is still an open question. MGN1703 is a synthetic immunomodulator acting as a TLR-9 agonist. Fifty-nine CRC patients were randomized 2:1 in IMPACT, a placebocontrolled Phase II trial testing maintenance with MGN1703 after a first-line treatment. The median PFS was longer in the experimental arm (HR: 0.56; CI: 95\% 0.29-1.08; $\mathrm{p}=0.07$ ) and nearly $10 \%$ of the patients experienced sustained PFS while on treatment [20]. Unfortunately, the trial was prematurely closed due to low recruitment, preventing the authors from drawing any definitive conclusions.

\section{Should all patients receive bevacizumab beyond progression?}

Retrospective registries (ARIES and BRITE) [21,22] and prospective studies (ML18147) [23] consistently reported survival benefits with the use of bevacizumab beyond disease progression. In the Phase III BEBYP trial, 184 patients who had failed a bevacizumab-based first-line treatment were randomized to receive second-line chemotherapy with or without bevacizumab. The trial was stopped early, as soon as the positive results of the ML18147 were diffused. Updated data of BEBYP were reported at the meeting [24]. Patients were stratified according to performance status (Eastern Cooperative Oncology Group performance scale: 0 vs 1-2), length of the chemotherapy-free interval ( $<3$ months or $>3$ months), and type of second-line chemotherapy. Oxaliplatin-based combinations were prescribed in $66 \%$ of the patients in both treatment arms. After a median follow-up of 22 months, the results confirmed the benefit in PFS (6.8 vs 5 months; HR: 0.72 ; 95\% CI: 0.54-0.97; $\mathrm{p}=0.029)$ for those maintained on bevacizumab. Subgroup analyses showed a consistent benefit in all subgroups, while OS data are still too immature to be analyzed. 


\section{Neurotoxicity \& neuroprotection: a step back?}

No measures can prevent the development of cumulative sensory neurotoxicity, which commonly leads to early discontinuation of oxaliplatin-based therapy. The use of intravenous calcium and magnesium $(\mathrm{CaMg})$ was associated with reduced sensory neurotoxicity, although this was observed in an underpowered NCCTG trial [25]. In the Phase III N08CB trial, 353 patients treated with oxaliplatin-based regimens were randomized to either $\mathrm{CaMg}$ before and after chemotherapy, placebo before and after chemotherapy or $\mathrm{CaMg}$ before and placebo after chemotherapy. No statistically significant reductions in cumulative neurotoxicity (European Organisation for Research and Treatment of Cancer patient self-report questionnaire assessing chemotherapy-induced peripheral neuropathy and Common Terminology Criteria for Adverse 4.0) were observed [26].

Once again, significant novel data focusing on colorectal cancer management have been presented at the 2013 World Congress in
Gastrointestinal Cancers. Not only do these data have a deep impact on a more accurate comprehension of tumor biology, but they also immediately translate into clinical practice advantages. In particular, the optimal choice of the upfront therapy in KRAS wild-type patients and the selection of the most appropriate treatment strategy will help colorectal cancer patients to reach an increased median survival.

\section{Financial \& competing interests disclosure \\ $C$ Cremolini has been an invited speaker for Bayer. F Loupakis has been an invited speaker for Roche, Amgen and Bayer. G Aprile has been an invited speaker for Roche, Merck and Amgen. C Cremolini, F Loupakis and $G$ Aprile were involved as investigators in some of the trials presented. The authors have no other relevant affiliations or financial involvement with any organiza- tion or entity with a financial interest in or financial conflict with the subject matter or materials discussed in the manuscript apart from those disclosed. \\ No writing assistance was utilized in the production of this manuscript.}

\section{References}

1. Heinemann V, von Weikersthal LF, Thomas Decker $\mathrm{T}$ et al. Randomized comparison of FOLFIRI plus cetuximab versus FOLFIRI plus bevacizumab as first-line treatment of KRAS wild-type metastatic colorectal cancer: German AIO study KRK-0306. Presented at: ASCO Annual Meeting. Chicago, IL, USA, 31 May-4 June 2013.

2. Modest M, Fischer von Weikersthal L, Stintzing $S$ et al. Folfiri plus cetuximab versus Folfiri plus bevacizumab as first-line treatment of KRAS-wild type metastatic colorectal cancer: German AIO Study KRK-0306 (FIRE-3). Ann. Oncol. 24(4) 1693-1699.

3. Rivera F, Karthaus M, Fasola G et al. KRAS/NRAS mutations in PEAK: a randomized Phase II study of 1st-line treatment with FOLFOX6 + panitumumab or bevacizumab for wild-type KRAS MCRC. Presented at: ESMO 15th World Congress on Gastrointestinal Cancer. Barcelona, Spain, 3-6 July 2013.

4. Mansmann U, Sartorius U, Laubender R, Giessen C, Esser R, Heinemann V. Quantitative analysis of the impact of deepness of response on post-progression survival time following first-line treatment in patients with mCRC. Presented at: ESMO 15th World Congress on Gastrointestinal Cancer. Barcelona, Spain, 3-6 July 2013.
5. Oliner KS, Douillard JY, Siena S et al. Analysis of KRAS/NRAS and BRAF mutations in the phase III PRIME study of panitumumab (pmab) plus FOLFOX versus FOLFOX as first-line treatment (tx) for metastatic colorectal cancer (mCRC). Presented at: 2013 ASCO Annual Meeting. Chicago, IL, USA, 31 May-4 June 2013.

6. Oliner K, Douillard JY, Siena $\mathrm{S}$ et al. Evaluation of $K R A S, N R A S$, and $B R A F$ mutations in PRIME: panitumumab with FOLFOX4 as first-line treatment in metastatic colorectal cancer. Presented at: ESMO 15th World Congress on Gastrointestinal Cancer. Barcelona, Spain, 3-6 July 2013.

7. Sommeijer DW, Shi Q, Meyers JP et al. Prognostic value of early objective tumor response (EOTR) to first-line systemic therapy in metastatic colorectal cancer (mCRC): individual patient data (IPD) meta-analysis of randomized trials from the ARCAD database. Presented at: 2013 ASCO Annual Meeting. Chicago, IL, USA, 31 May-4 June 2013.

8. Adam R, Bridgewater J, Chau I et al. Randomized Phase II study (OLIVIA) of bevacizumab plus mFOLFOX6 or FOLFOXIRI in patients with initially unresectable colorectal cancer liver metastases. Presented at: ESMO 15th World Congress on Gastrointestinal Cancer. Barcelona, Spain, 3-6 July 2013.

9. Cremolini C, Loupakis F, Masi G et al. FOLFOXIRI/bevacizumab versus
FOLFIRI/bevacizumab as first-line treatment in unresectable metastatic colorectal cancer: results of Phase III TRIBE trial by GONO Group. Presented at: ESMO 15th World Congress on Gastrointestinal Cancer. Barcelona, Spain, 3-6 July 2013.

10. Aprile G, Ferrari L, Fontanella C, Puglisi F. Bevacizumab in older patients with advanced colorectal or breast cancer. Crit. Rev. Oncol. Hematol. 87(1), 41-54 (2013).

11. Cunningham D, Lang I, Marcuello E et al.; for the AVEX study investigators. Bevacizumab plus capecitabine versus capecitabine alone in elderly patients with previously untreated metastatic colorectal cancer (AVEX): an openlabel, randomised Phase 3 trial. Lancet Oncol. 14(11), 1077-1085 (2013).

12. Saunders M, Lang I, Marcuello E et al. Results according to age in AVEX, a randomized Phase III trial of bevacizumab with capecitabine for elderly patients with MCRC. Presented at: ESMO 15th World Congress on Gastrointestinal Cancer. Barcelona, Spain, 3-6 July 2013.

13. Van Cutsem E, Tabernero J, Lakomy R et al. Addition of aflibercept to fluorouracil, leucovorin, and irinotecan improves survival in a Phase III randomized trial in patients with metastatic colorectal cancer previously treated with an oxaliplatin-based regimen. J. Clin. Oncol. 30(28), 3499-3506 (2012).

14. Ruff P, Ferry D, Papamichael D et al. Observed benefit of aflibercept in MCRC 
patients $>65$ years old: results of a prespecified age-based analysis of the VELOUR study. Presented at: ESMO 15th World Congress on Gastrointestinal Cancer. Barcelona, Spain, 3-6 July 2013.

15. Díaz-Rubio E, Gómez-España A, Massutí B et al. First-line XELOX plus bevacizumab followed by XELOX plus bevacizumab or single-agent bevacizumab as maintenance therapy in patients with metastatic colorectal cancer: the Phase III MACRO TTD study. Oncologist 17, 15-25 (2012).

16. Tournigand C, Samson B, Scheithauer W et al. mFOLFOX-bevacizumab or XELOXbevacizumab then bevacizumab (B) alone or with erlotinib (E) in first-line treatment of patients with metastatic colorectal cancer (mCRC): interim safety analysis of DREAM study. J. Clin. Oncol. 27(Suppl. 15), 4077 (2009).

17. Wasan H, Adams RA, Wilson RH et al. Intermittent chemotherapy (CT) plus continuous or intermittent cetuximab (C) in the first-line treatment of advanced colorectal cancer (aCRC): Results of the two-arm Phase II randomized MRC COIN-b trial. J. Clin. Oncol. 30(Suppl. 4), Abstract 536 (2012).

18. Koopman M, Simkens LH, Ten Tije AJ et al. Maintenance treatment with capecitabine and bevacizumab versus observation after induction treatment with chemotherapy and bevacizumab in metastatic colorectal cancer (mCRC): the Phase III CAIRO3 study of the Dutch Colorectal Cancer Group (DCCG). Presented at: 2013 ASCO Annual Meeting. Chicago, IL, 31 May-4 June 2013.

19. Koeberle D, Betticher D, von Moos R et al. Bevacizumab continuation versus no continuation after first-line chemo-bevacizumab therapy in patients with metastatic colorectal cancer: a Phase III non-inferiority trial. Presented at: ESMO 15th World Congress on Gastrointestinal Cancer. Barcelona, Spain, 3-6 July 2013.

20. Schmoll HJ, Riera-Knorrenschild J, Kroening $\mathrm{H}$ et al. Updated results of the randomized Phase 2 IMPACT trial: maintenance with TLR-9 agonist MGN1703 vs placebo in patients with metastatic colorectal carcinoma (MCRC). Presented at: ESMO 15th World Congress on Gastrointestinal Cancer. Barcelona, Spain, 3-6 July 2013.

21. Bendell JC, Bekaii-Saab TS, Cohn AL et al. Treatment patterns and clinical outcomes in patients with metastatic colorectal cancer initially treated withFOLFOX-bevacizumab or FOLFIRI-bevacizumab: results from ARIES, a bevacizumab observational cohort study. Oncologist 17(12), 1486-1495 (2012).

22. Kozloff MF, Berlin J, Flynn PJ et al. Clinical outcomes in elderly patients with metastatic colorectal cancer receiving bevacizumab and chemotherapy: results from the BRITE observational cohort study. Oncology 78, 329-339 (2010).

23. Bennouna J, Sastre J, Arnold D et al.; ML18147 Study Investigators. Continuation of bevacizumab after first progression in metastatic colorectal cancer (ML18147): a randomised Phase 3 trial. Lancet Oncol. 14(1), 29-37 (2013).

24. Salvatore L, Masi G, Loupakis F et al. Bevacizumab beyond progression in metastatic colorectal cancer patients receiving a first-line treatment containing bevacizumab: update of BEBYP trial by GONO. Presented at: ESMO 15th World Congress on Gastrointestinal Cancer. Barcelona, Spain, 3-6 July 2013.

25. Grothey A, Nikcevich DA, Sloan JA et al. Intravenous calcium and magnesium for oxaliplatin-induced sensory neurotoxicity in adjuvant colorectal cancer: NCCTG N04C7. J. Clin. Oncol. 29(4), 421-427 (2011).

26. Grothey A, Qin R, Dakhil S et al. Phase III randomized, placebo-controlled, double-blind study of intravenous calcium/magnesium (CAMG) to prevent oxaliplatin-induced sensory neurotoxicity (SNT), N08CB: an alliance for clinical trial in oncology study 1. Presented at: ESMO 15th World Congress on Gastrointestinal Cancer. Barcelona, Spain, 3-6 July 2013. 